\title{
Searching for Cosmological Preferred Axis using cosmographic approach
}

\author{
Amin.Salehi and Mohammad Reza Setare* \\ Department of Physics, Lorestan University, Lorestan, Irant and \\ Department of Science, Campus of Bijar, \\ University of Kurdistan, Bijar, Iran.
}

\begin{abstract}
Recent released Planck data and other astronomical observations show that the universe may be anisotropic on large scales. This hints a cosmological privileged axis in our anisotropic expanding universe. This paper proceeds a modified redshift in anisotropic cosmological model as $1+\tilde{z}(t, \hat{\mathbf{p}})=$ $\frac{a\left(t_{0}\right)}{a(t)}(1-A(\hat{\mathbf{n}} \cdot \hat{\mathbf{p}}))$ (where $A$ is the magnitude of anisotropy,$\hat{\mathbf{n}}$ is the direction of privileged axis, and $\hat{\mathbf{p}}$ is the direction of each SNe Ia sample to galactic coordinates) along with anisotropic parameter $\delta=$ $\frac{A(\hat{\mathbf{n}} \cdot \hat{\mathbf{p}})}{1+A(\hat{\mathbf{n}} \cdot \hat{\mathbf{p}})}$. The luminosity distance is expanded with model-independent cosmographic parameters as a function of modified redshift $\tilde{z}$. As the transformation matrix $M(n \times n)$ is obtained to convert the Taylor series coefficients of isotropic luminosity distance to corresponding anisotropic parameters. These results culminate the magnitude of anisotropy about $|A| \simeq 10^{-3}$ and the direction of preferred axis as $(l, b)=\left(297_{+34}^{-34}, 3_{+28}^{-28}\right)$, which are consistent with other studies in $1-\sigma$ confidence level.
\end{abstract}

PACS numbers: 98.80.Es; 98.80.Bp; 98.80.Cq

Keywords: Extended gravity; stability; attractor; Cosmography

*Electronic address: salehi.a@lu.ac.ir

$\dagger$ Electronic address: rezakord@ipm.ir 


\section{INTRODUCTION}

According to cosmological principle, as one of the basic assumptions of modern cosmology, universe is homogeneous and isotropic on scales larger than a few hundred Mpc. Indeed, this assumption is consistent with former astronomical observations such as the Cosmic Microwave Background (CMB) radiation data from the Wilkinson Microwave Anisotropy Probe (WMAP) [1]-[3], and data from type Ia supernovae, such as those collected in the so-called Union [4] , Union2 [5] compilations . However, some challenges are reported to the cosmological principle in recent years. For example, the large-scale

alignments of the quasar polarization vectors [6]-[7], the large-scale bulk flow [8]-[10], the alignments of low multipoles in CMB angular power spectrum[11]-[15], the spatial variation of fine-structure constant [16]-[21], the CMB hemispherical asymmetry observed by WMAP [22]-[23] and Planck satellite [24], Dark Energy Dipole[25]-[28], and other effects [29]-[30].

There are many models, in the theoretical aspect, which have been proposed to resolve the asymmetric anomaly of these data. Several groups have applied the hemisphere comparison method to study the anisotropy of $\Lambda C D M, w C D M$ and the dark energy model with $C P L$ parametrization. So that, the supernova data and the corresponding cosmic accelerations on several pairs of opposite hemispheres have been used to search for maximally asymmetric pair and a statistically significant preferred axis [34]. Ref [33] have applied the hemisphere comparison method to the standard $\Lambda C D M$ model and found that the hemisphere of maximum accelerating expansion is in the direction $\left.(l ; b)=309_{+23}^{-3}, 18_{+11}^{-10}\right)$ with Union2 data. Also the Ref [36] tests the isotropy of the expansion of the Universe by estimating the hemispherical anisotropy of supernova type Ia (SN Ia) Hubble diagrams at low redshifts $(z<0.2)$ and found a maximal hemispheric asymmetry towards a direction close to the equatorial poles with Hubble anisotropy of $\frac{\Delta H}{H}=0.026$. The anisotropic cosmological model is investigated by Ref.[48] in the Randers spacetime, to determine the privilege direction of $(l, b)=306,-18)$.

A potpourri list of pertinent studies is provided in Table I. Ref. [35], have taken the deceleration parameter $q_{0}$ as the diagnostic to quantify the anisotropy level in the $\omega C D M$ model. The authors of Ref.[28] constructed a direction-dependent dark energy model 
TABLE I: Incomplete list of pervious studies

\begin{tabular}{|c|c|c|c|}
\hline 1 & models & Equation & Ref \\
\hline 1 & scalar perturbation & $\left(d_{L}=(1+z) \frac{c}{H_{0}} \int_{0}^{z} \frac{(1-d \cos \theta) d z}{\sqrt{\Omega_{m 0}(1+z)^{3}+1-\Omega_{m 0}-\frac{4 d \cos \theta(1+x)^{5}}{3 H_{0}^{2} d_{L 0}^{2}}}}\right)$ & [39], ,37] \\
\hline 2 & Anisotropic $d_{L}$ in the Finslerian space-time & $\left(d_{L}=(1+z) \frac{c}{H_{0}} \int_{0}^{z} \frac{(1-d \cos \theta)^{-1} d z}{\sqrt{\Omega_{m}\left(\frac{1-d \cos \theta}{1+z}\right)^{-3}+\Omega_{\Lambda}}}\right)$ & [48] \\
\hline 3 & effect of peculiar velocities on $d_{L}$ & $\frac{\Delta d_{L}}{d_{L}}=\hat{n} \cdot\left[\vec{v}_{p e c}-\left(\vec{v}_{p e c}-\vec{v}_{o b s}\right) \cdot \frac{(1+z)^{2}}{H(z) d_{L}}\right]$ & [42]- $[46]$ \\
\hline 4 & wind scenario to the bulk flow & $\left(d_{L}=(1+z) \int_{0}^{t} \frac{d t^{\prime}}{a\left(t^{\prime}\right)}(1+d \cos \theta)=\bar{d}_{L}(1+d \cos \theta)\right)$ & {$[40]$} \\
\hline 5 & dipole + monopole fit approach & $\left(\frac{\Delta \mu}{\mu}=d \cos \theta+m\right)$ & {$[38],[33],[37]$} \\
\hline 6 & hemisphere comparison method & $\frac{\Delta \Omega_{0 m}}{\Omega_{0 m}}=2\left(\frac{\Omega_{0 m, u}-\Omega_{0 m, d}}{\Omega_{0 m, u}+\Omega_{0 m, d}}\right)$ & {$[38],[28]$} \\
\hline 7 & hemisphere comparison method & $\frac{\Delta q_{0}}{\overline{q_{0}}}=2\left(\frac{q_{0, u}-q_{0, d}}{q_{0, u}+q_{0, d}}\right)$ & [35] \\
\hline $\begin{array}{l}8 \\
9\end{array}$ & $\begin{array}{l}\text { luminosity-distance } \\
\text { in ellipsoidal universe }\end{array}$ & $\begin{array}{c}d_{L}(z, \theta)=\frac{1+z}{H 0} \int_{A(z)}^{1} \frac{d A}{A^{2} \bar{H}} \frac{\left(1-e^{2}\right)^{1 / 6}}{\left(1-e^{2} \cos \theta\right)^{1 / 2}} \\
1+z=\frac{1}{A} \frac{\left(1-e^{2} \sin \theta\right)^{1 / 2}}{\left(1-e^{2}\right)^{1 / 3}}\end{array}$ & {$[26]$} \\
\hline 10 & $\alpha($ dipole + monopole $)$ fit approach & $\frac{\Delta \alpha}{\alpha}=A \cos \theta+B$ & {$[33],[18]$} \\
\hline 11 & measured (perturbed) luminosity-distance & $D_{L}=\left(1+2 \hat{n} \cdot \overrightarrow{v_{s}}\right) D_{0 L}, v_{s}=$ peculiar velocities & {$[41]$} \\
\hline 12 & dipole fit approach & $\frac{d_{L}(z)-d_{L}^{0}(z)}{d_{L}^{0}(z)}=g(z)(\hat{z} \cdot \hat{n})$ & [35] \\
\hline
\end{tabular}

based on the isotropic background described by the $\Lambda C D M, \omega C D M$ and $C P L$ models and employed the Union2 dataset to constrain the anisotropy direction and strength of modulation. They found the best-fitting value of the maximum deviation direction from the isotropic background, which was not sensitive to the details of isotropic dark energy models. Ref.[37] have studied dipolar anisotropic expansion with cosmographic parameters, and found the preffered direction of $\left(l=309^{\circ}, b=-8.6^{\circ}\right)$. The athours of [38] choosed two simple cosmological models, $\Lambda C D M$ and $\omega C D M$ for the hemisphere comparison approach, and $\Lambda C D M$ for the dipole fit. In the first approach, they used the matter density and the equation of state of dark energy as the diagnostic qualities in the $\Lambda C D M$ and $\omega C D M$, respectively. In the second method, they employed distance modulus as the diagnostic quality in $\Lambda C D M$, and found the preferred direction of $\left(l=307^{\circ}, b=-14^{\circ}\right)$.

Here, we investigate the anisotropy expansion of the universe using the model-independent cosmography method. The cosmography method brings forward a part of cosmology which does not postulate any precedential cosmological model. Thus, it can be thought as a model- 
independent way to fix the constraints on the universe's dynamics at late times through the use of a set of parameters; namely the cosmographic set (CS) [52]-[53]. The outline of the paper is as follows. The cosmographic apparatus is reviewed in Section. II. The luminosity distance redshift relation in the anisotropic Universe will be extracted in Section III. The acquisition way of the matrix $M(n \times n)$ is introduced to convert the Taylor series coefficients of isotropy luminosity distance to anisotropy factors of luminosity distance. The numerical results are given in Section. IV and in order to discuss and compare with other works. Arguments are given in section $\mathrm{V}$.

\section{THE COSMOGRAPHIC APPARATUS}

One of the basic relations in modern cosmology and cosmography is the luminosity distance redshift $d_{L}(z)$ used in the definition of redshift in an isotropic Universe

$$
1+z=\frac{a\left(t_{0}\right)}{a(t)}
$$

The following relation is also retained from the cosmographic approach [52]-[53];

$$
d_{L}(z)=\frac{c z}{H_{0}}\left\{\mathcal{D}_{L}^{0}+\mathcal{D}_{L}^{1} z+\mathcal{D}_{L}^{2} z^{2}+\mathcal{D}_{L}^{3} z^{3}+\mathcal{D}_{L}^{4} z^{4}+O\left(z^{5}\right)\right\}
$$

In which;

$$
\begin{aligned}
\mathcal{D}_{L}^{0} & =1 \\
\mathcal{D}_{L}^{1} & =-\frac{1}{2}\left(-1+q_{0}\right) \\
\mathcal{D}_{L}^{2} & =-\frac{1}{6}\left(1-q_{0}-3 q_{0}^{2}+j_{0}+\frac{k c^{2}}{H_{0}^{2} a_{0}^{2}}\right) \\
\mathcal{D}_{L}^{3} & =\frac{1}{24}\left(2-2 q_{0}-15 q_{0}^{2}-15 q_{0}^{3}+5 j_{0}+10 q_{0} j_{0}+s_{0}+\frac{2 k c^{2}\left(1+3 q_{0}\right)}{H_{0}^{2} a_{0}^{2}}\right) \\
\mathcal{D}_{L}^{4} & =\frac{1}{120}\left[-6+6 q_{0}+81 q_{0}^{2}+165 q_{0}^{3}+105 q_{0}^{4}-110 q_{0} j_{0}-105 q_{0}^{2} j_{0}-15 q_{0} s_{0}+\right. \\
& \left.-27 j_{0}+10 j_{0}^{2}-11 s_{0}-l_{0}-\frac{5 k c^{2}\left(1+8 q_{0}+9 q_{0}^{2}-2 j_{0}\right)}{a_{0}^{2} H_{0}^{2}}\right]
\end{aligned}
$$

Where the cosmographic parameters are defined as;

$$
\left.H(t) \equiv+\frac{1}{a} \frac{d a}{d t}, \quad q(t) \equiv-\frac{1}{a} \frac{d^{2} a}{d t^{2}} \frac{1}{H^{2}} \quad, \quad j(t) \equiv+\frac{1}{a} \frac{d^{3} a}{d t^{3}} \frac{1}{H^{3}}, \quad s(t) \equiv+\frac{1}{a} \frac{d^{4} a}{d t^{4}} \frac{1}{H^{4}}, \quad l(t) \equiv+\frac{1}{a} \frac{d^{5} a}{d t^{5}} \frac{1}{H^{5}} 8\right)
$$




\section{THE LUMINOSITY DISTANCE REDSHIFT RELATION IN THE ANISOTROPIC UNIVERSE}

In the anisotropic cosmological model, the redshift of an object located in the $\hat{\mathbf{p}}$ direction of each SNe Ia sample to galactic coordinates and at time t can be modified as

$$
1+\tilde{z}(t, \hat{\mathbf{p}})=\frac{a\left(t_{0}\right)}{a(t)}(1-A(\hat{\mathbf{n}} \cdot \hat{\mathbf{p}}))
$$

where $|A|<<1$ represents the magnitude of anisotropy of the universe and $\hat{\mathbf{n}}$ is the direction of the privileged axis

In the situation, by succession of $\tilde{z}$ rather than $z$ in equation (2), the anisotropy luminosity distance will be equal to,

$$
d_{L}(\tilde{z})=\frac{c \tilde{z}}{H_{0}}\left\{\mathcal{D}_{L}^{0}+\mathcal{D}_{L}^{1} \tilde{z}+\mathcal{D}_{L}^{2} \tilde{z}^{2}+\mathcal{D}_{L}^{3} \tilde{z}^{3}+\mathcal{D}_{L}^{4} \tilde{z}^{4}+O\left(\tilde{z}^{5}\right)\right\}
$$

Here we suppose that $H_{0}$ is constant. However angular anisotropy of $H_{0}$ has been studied in several works (see for example[54]). Using equations (9) and (19), and since $A$ is a very small magnitude,

$$
1+z=\frac{1+\tilde{z}}{1-A(\hat{\mathbf{n}} \cdot \hat{\mathbf{p}})}=(1+\tilde{z})(1+A(\hat{\mathbf{n}} \cdot \hat{\mathbf{p}}))
$$

where for $A \ll 1$, we have used $\left(\frac{1}{1-x} \simeq 1+x\right)$. Therefore, the equation takes the modified redshift $\tilde{z}$ in the following form,

$$
\tilde{z} \simeq \frac{1+z}{1+A(\hat{\mathbf{n}} \cdot \hat{\mathbf{p}})}-1 \simeq \frac{z-A(\hat{\mathbf{n}} \cdot \hat{\mathbf{p}})}{1+A(\hat{\mathbf{n}} \cdot \hat{\mathbf{p}})} \simeq \frac{z}{1+A(\hat{\mathbf{n}} \cdot \hat{\mathbf{p}})}-\frac{A(\hat{\mathbf{n}} \cdot \hat{\mathbf{p}})}{1+A(\hat{\mathbf{n}} \cdot \hat{\mathbf{p}})} \simeq z-\frac{A(\hat{\mathbf{n}} \cdot \hat{\mathbf{p}})}{1+A(\hat{\mathbf{n}} \cdot \hat{\mathbf{p}})}
$$

where for very small value of $A$, we have $\operatorname{used}(1+A(\hat{\mathbf{n}} \cdot \hat{\mathbf{p}}) \simeq 1)$ and consequently $\left(\frac{z}{1+A(\hat{\mathbf{n}} \cdot \hat{\mathbf{p}})} \simeq\right.$ $z)$. By considering $\delta=\frac{A(\hat{\mathbf{n}} \cdot \hat{\mathbf{p}})}{1+A(\hat{\mathbf{n}} \cdot \hat{\mathbf{p}})}$ and substituting $(\tilde{z}=z-\delta)$ into equation (10), we can rewrite this equation as

$d_{L}(\tilde{z})=\frac{c(z-\delta)}{H_{0}}\left\{\mathcal{D}_{L}^{0}+\mathcal{D}_{L}^{1}(z-\delta)+\mathcal{D}_{L}^{2}(z-\delta)^{2}+\mathcal{D}_{L}^{3}(z-\delta)^{3}+\mathcal{D}_{L}^{4}(z-\delta)^{4}+\ldots \ldots \mathcal{D}_{L}^{n}(z-\delta)^{n}(\mathfrak{\}} 3)\right.$

By expanding $(z-\delta)^{m}$ using binomial theorem,

$$
(x+y)^{m}=\sum_{k=0}^{m}\left(\begin{array}{c}
m \\
k
\end{array}\right) x^{m-k} y^{k}
$$

The luminosity distance $d_{L}$ will be obtained in terms of $z$ with new constant coefficients $\mathcal{D}_{L}^{m}$ as 


$$
\left.d_{L}(\tilde{z})=\frac{c z}{H_{0}}\left\{\mathcal{D}_{L}^{0}+\mathcal{\mathcal { D }}_{L}^{1} z+\dot{\mathcal{D}}_{L}^{2} z^{2}+\dot{\mathcal{D}}_{L}^{3} z^{3}+\dot{\mathcal{D}}_{L}^{4} z^{4}+\ldots \mathcal{\mathcal { D }}_{L}^{n} z^{n}\right)\right\}
$$

Where, the anisotropic coefficients can be obtain by expanding the luminosity distance to the nth order of redshift $\tilde{z}$, from the corresponding isotropic factors through the transformation matrix $M$ as,

$$
\left(\begin{array}{c}
\mathcal{D}_{L}^{n} \\
\mathcal{D}_{L}^{n-1} \\
\mathcal{D}_{L}^{n-2} \\
\cdot \\
\cdot \\
\mathcal{D}_{L}^{0}
\end{array}\right)=\mathrm{M}\left(\begin{array}{c}
\mathcal{D}_{L}^{n} \\
\mathcal{D}_{L}^{n-1} \\
\mathcal{D}_{L}^{n-2} \\
\cdot \\
\cdot \\
\mathcal{D}_{L}^{0}
\end{array}\right)
$$

So that, the matrix $M$ would be,

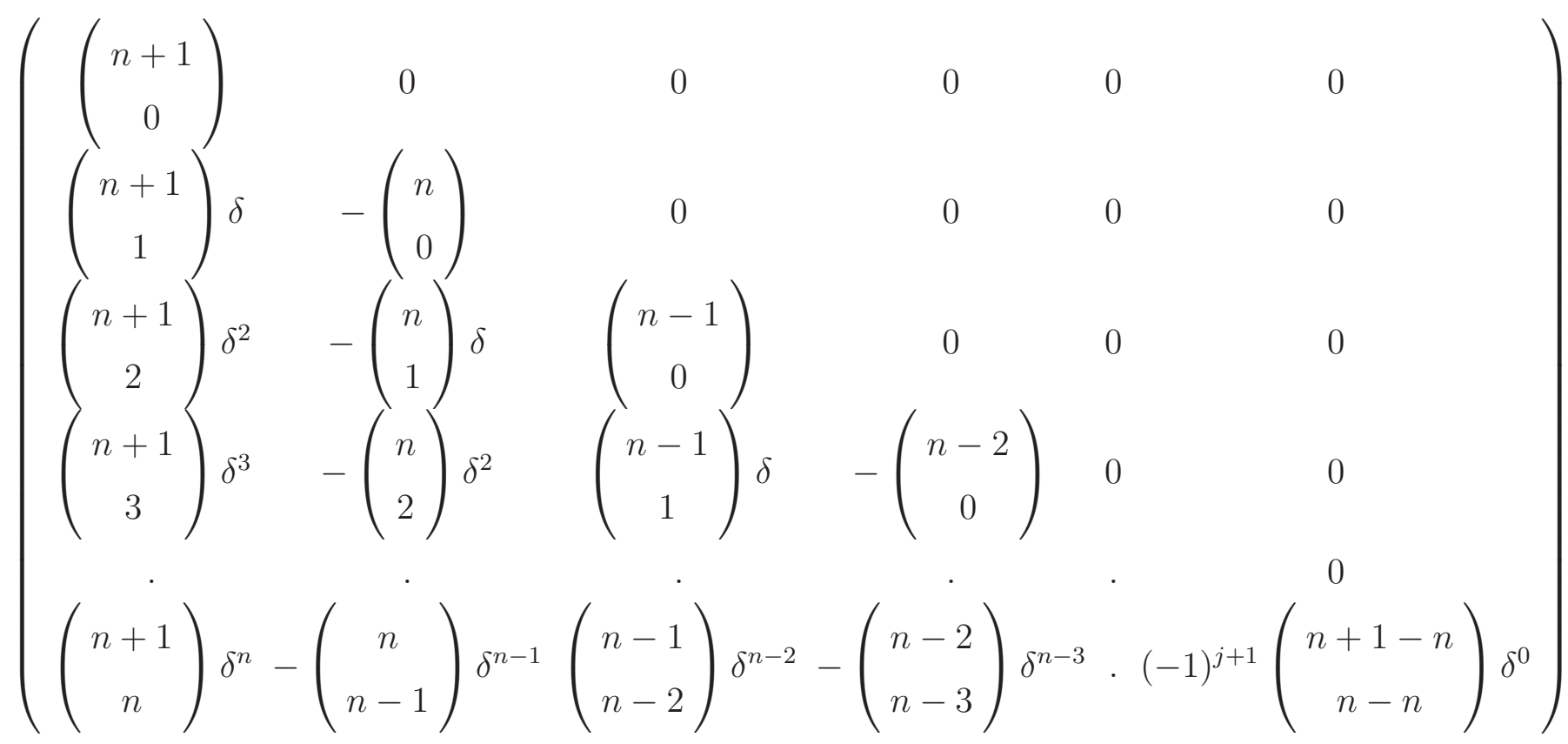


Neglecting higher order terms of $\delta^{n}$ with $n>1$,

$$
\begin{aligned}
\mathcal{D}_{L}^{0} & =\left(\mathcal{D}_{L}^{0}-2 \mathcal{D}_{L}^{1} \delta\right) \\
\mathcal{D}_{L}^{1} & =\left(\mathcal{D}_{L}^{1}-3 \mathcal{D}_{L}^{2} \delta\right. \\
\mathcal{D}_{L}^{2} & =\left(\mathcal{D}_{L}^{2}-4 \mathcal{D}_{L}^{3} \delta\right) \\
\mathcal{D}_{L}^{3} & =\left(\mathcal{D}_{L}^{3}-5 \mathcal{D}_{L}^{4} \delta\right) \\
\cdot & = \\
\cdot & = \\
\mathcal{D}_{L}^{n-1} & =\left(\mathcal{D}_{L}^{n-1}-(n+1) \mathcal{D}_{L}^{n+1} \delta\right) \\
\mathcal{D}_{L}^{n} & =\mathcal{D}_{L}^{n}
\end{aligned}
$$

For example, for series expansion of $d_{L}(\tilde{z})$ to the 5 th order in redshift $\tilde{z}$, we have

$$
d_{L}(\tilde{z})=\frac{c z}{H_{0}}\left\{\dot{\mathcal{D}}_{L}^{0}+\dot{\mathcal{D}}_{L}^{1} z+\dot{\mathcal{D}}_{L}^{2} z^{2}+\dot{\mathcal{D}}_{L}^{3} z^{3}+\dot{\mathcal{D}}_{L}^{4} z^{4}+O\left(z^{5}\right)\right\}
$$

where

$$
\begin{aligned}
\mathcal{D}_{L}^{0} & =\left(\mathcal{D}_{L}^{0}-2 \mathcal{D}_{L}^{1} \delta+3 \mathcal{D}_{L}^{2} \delta^{2}-4 \mathcal{D}_{L}^{3} \delta^{3}+5 \mathcal{D}_{L}^{4} \delta^{4}\right) \\
\mathcal{D}_{L}^{1} & =\left(\mathcal{D}_{L}^{1}-3 \mathcal{D}_{L}^{2} \delta+6 \mathcal{D}_{L}^{3} \delta^{2}-10 \mathcal{D}_{L}^{4} \delta^{3}\right) \\
\mathcal{D}_{L}^{2} & =\left(\mathcal{D}_{L}^{2}-4 \mathcal{D}_{L}^{3} \delta+10 \mathcal{D}_{L}^{4} \delta^{2}\right) \\
\mathcal{D}_{L}^{3} & =\left(\mathcal{D}_{L}^{3}-5 \mathcal{D}_{L}^{4} \delta\right) \\
\mathcal{D}_{L}^{4} & =\mathcal{D}_{L}^{4}
\end{aligned}
$$

Accordingly, the theoretical distance modulus $\mu_{t h}(\tilde{z})$ can be defined as follows,

$$
\mu_{t h}(\tilde{z})=5 \log _{10} d_{L}(\tilde{z})+42.384-5 \log _{10} h_{0}
$$

\section{NUMERICAL CONSTRAINTS FROM UNION2 DATA}

The Union2 SnIa dataset [5] is a compilation consisting of 557 SNe Ia with the redshift range of $\mathrm{z}=[0: 015 ; 1: 4]$. The angular distribution of the Union 2 dataset in galactic coordinates, has been shown in Fig. 2. The Union2 data along with directions as presented in Ref. [31] include the SnIa name, the redshift in the CMB rest frame, the distance modulus 
and its uncertainties (that involves both of the observational and the intrinsic magnitude scatter). They also include the equatorial coordinates (right ascension and declination) of each SnIa. It is straightforward to convert these coordinates to galactic coordinates or to usual spherical coordinates $(\theta, \phi)$ in the equatorial or galactic systems[32]. Here, we have converted the equatorial coordinates of each supernova to the galactic coordinates. The Cartesian coordinates of the unit vectors $p_{i}$ can also be found corresponding to each quasar with galactic coordinates $(l, b)$. Since,

$$
\hat{p}_{i}=\cos \left(l_{i}\right) \sin \left(b_{i}\right) \hat{i}+\sin \left(l_{i}\right) \sin \left(b_{i}\right) \hat{j}+\cos \left(b_{i}\right) \hat{k}
$$

The direction of the privileged axis can also be written as,

$$
\hat{n}=\cos (l) \sin (b) \hat{i}+\sin (l) \sin (b) \hat{j}+\cos (b) \hat{k}
$$

In this right, one can use the Union2 compilation to constrain the parameters of model and determine the direction of preferred axis of universe, in the framework of following steps:

- Find the best value for the cosmographic parameters $\left\{q_{0}, j_{0}, s_{0}, l_{0}, ..\right\}$ in isotropic background to find the best value for expansion's coefficients of isotropic luminosity distance $\left\{\mathcal{D}_{L}^{0}, \mathcal{D}_{L}^{1}, \mathcal{D}_{L}^{2} \ldots \mathcal{D}_{L}^{n}\right\}$

- Find the coefficients of anisotropic luminosity distance $\left\{\mathcal{D}_{L}^{0}, \mathcal{D}_{L}^{1}, \mathcal{D}_{L}^{2} \ldots . \mathcal{D}_{L}^{n}\right\}$ using the transformation matrix $M$.

- Find the anisotropic parameters $(l, b, A)$ and $\delta$ by doing the least $\chi^{2}$ fit to the Union2 data for equation (30).

In order to find the best value for the isotropic and anisotropic parameters of the model using SnIa data and examine the sensitivity of the model with order of luminosity distance expansion, we perform the $\chi^{2}$ method for the first, second , third and fourth order of the expansion. Thus we get the $M_{1}, M_{2}, M_{3}$ and $M_{4}$ as follows;

$$
\begin{gathered}
M_{1}: d_{L}(\tilde{z})=\frac{c z}{H_{0}}\left\{\mathcal{D}_{L}^{0}+\mathcal{D}_{L}^{1} z\right\} \\
M_{2}: d_{L}(\tilde{z})=\frac{c z}{H_{0}}\left\{\mathcal{D}_{L}^{0}+\mathcal{D}_{L}^{1} z+\mathcal{D}_{L}^{2} z^{2}\right\} \\
M_{3}: d_{L}(\tilde{z})=\frac{c z}{H_{0}}\left\{\dot{\mathcal{D}}_{L}^{0}+\mathcal{D}_{L}^{1} z+\dot{\mathcal{D}}_{L}^{2} z^{2}+\mathcal{D}_{L}^{3} z^{3}\right\} \\
M_{4}: d_{L}(\tilde{z})=\frac{c z}{H_{0}}\left\{\mathcal{D}_{L}^{0}+\mathcal{D}_{L}^{1} z+\mathcal{D}_{L}^{2} z^{2}+\mathcal{D}_{L}^{3} z^{3}+\dot{\mathcal{D}}_{L}^{4} z^{4}\right\}
\end{gathered}
$$


Using the maximum likelihood method (i.e. minimizing),

$$
\chi^{2}\left(h_{0}, q_{0}, j_{0}, \ldots\right)=\sum_{i=1}^{557} \frac{\left[\mu^{o b s}\left(z_{i}\right)-\mu^{t h}\left(z_{i} \mid h_{0}, q_{0}, j_{0}, \ldots\right]^{2}\right.}{\sigma^{2}\left(z_{i}\right)} .
$$

we can obtain the best value for cosmographic parameters in isotropic background.Table (II) shows these values for in isotropic background in $1-\sigma$ confidence level for $M_{1}$ to $M_{4}$ cases.Also the corresponding likelihood distribution and confidence level for these parameters have been shown in Figs (1) to (6).

It is interesting to note that, there is a considerable difference between the best value of cosmographic parameters $\left\{q_{0}, h_{0}\right\}$ in the $M_{1}$ case with higher orders $\left\{M_{2}, M_{3}, M_{4} ..\right\}$, however the model is not very sensitive to order of luminosity distance expansion for higher orders (see figs (1) to (6) and table II).

Using the best value of cosmographic in isotropic background and the maximum likelihood method (i.e. minimizing),

$$
\chi^{2}(A, l, b)=\sum_{i=1}^{557} \frac{\left[\mu^{o b s}\left(z_{i}\right)-\mu^{t h}\left(\tilde{z}_{i} \mid A, l, b\right)\right]^{2}}{\sigma^{2}\left(z_{i}\right)} .
$$
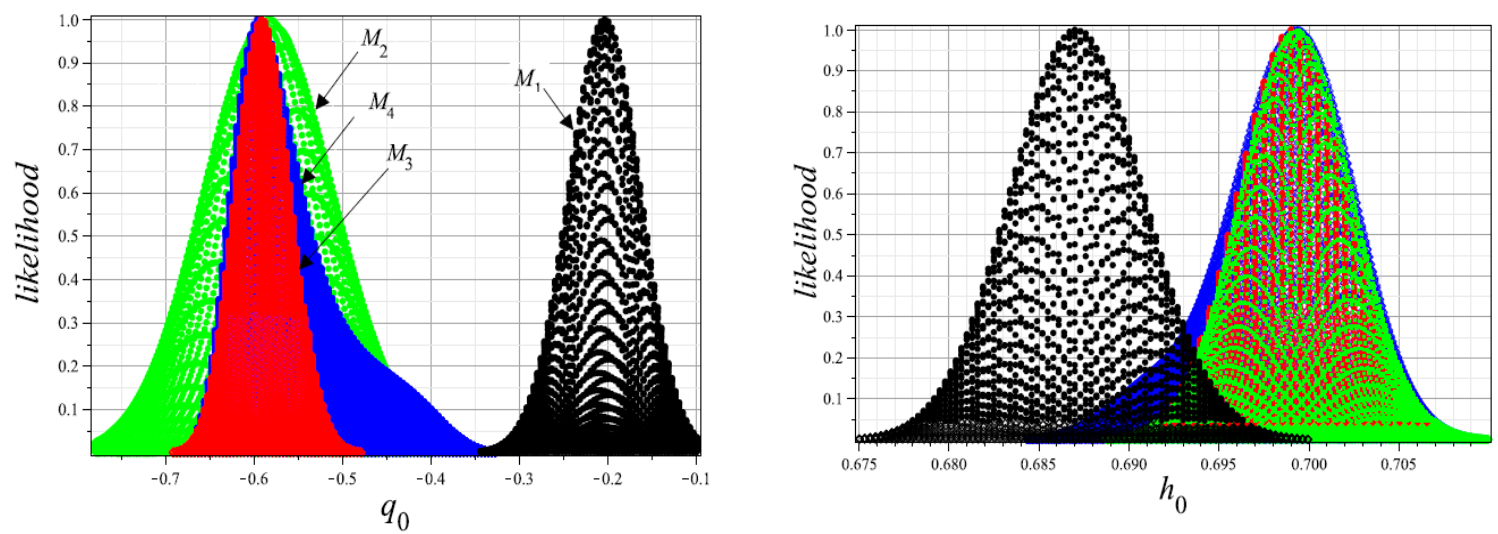

Fig. 1: The 1-dim likelihood for parameters $q_{0}$ and $h_{0}$ in $M_{1}, M_{2}, M_{3}$ and $M_{4}$ cases.

The black,green,red and blue colors represent the $M_{1}, M_{2}, M_{3}$ and $M_{4}$ cases respectively 

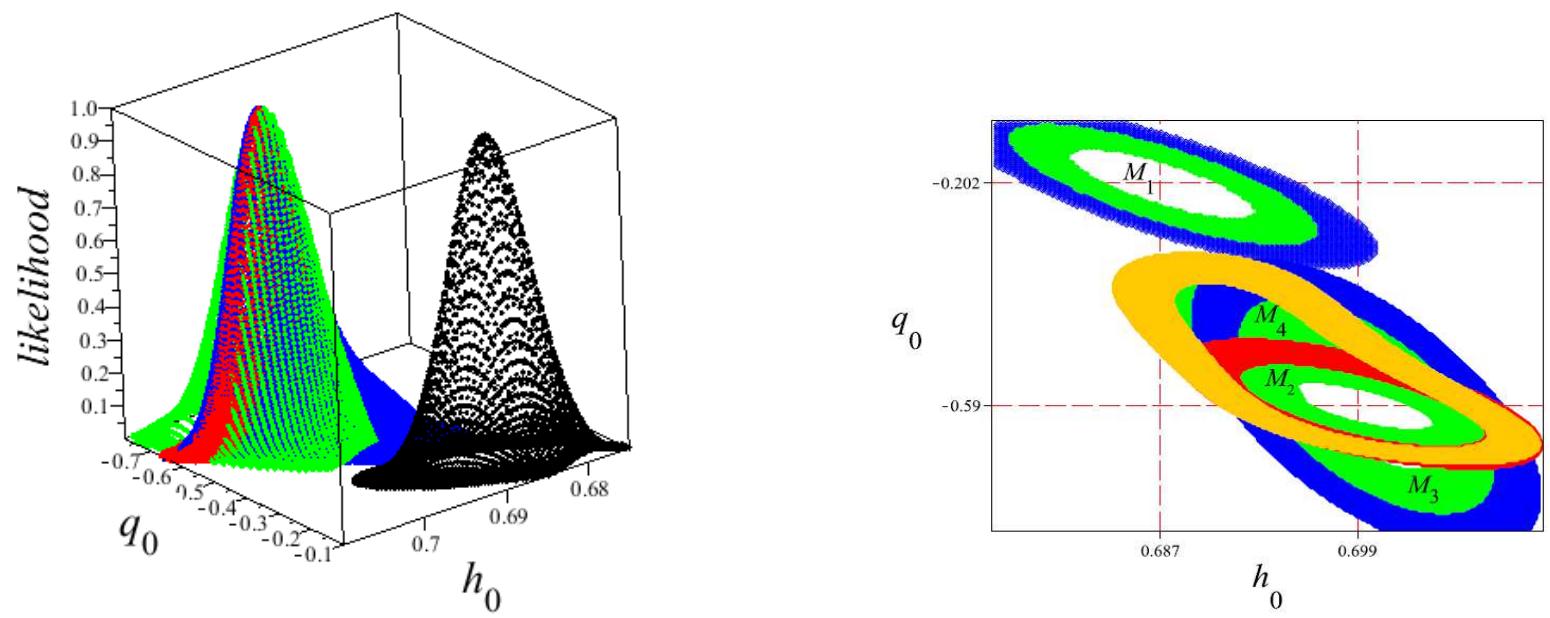

Fig. 2: The 2-dim likelihood and confidence level for parameters $q_{0}$ and $h_{0}$ in $M_{1}, M_{2}, M_{3}$ and $M_{4}$ cases.
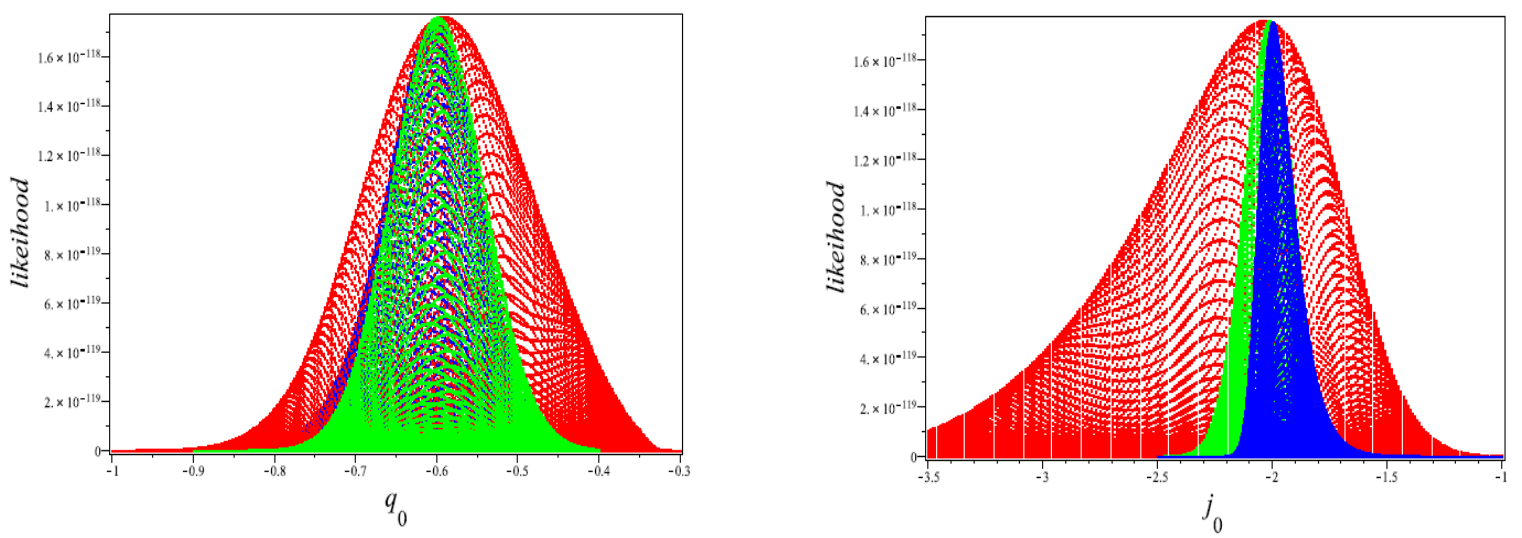

Fig. 3: The 1-dim likelihood for parameters $q_{0}$ and $j_{0}$ in $M_{2}, M_{3}$ and $M_{4}$ cases. 

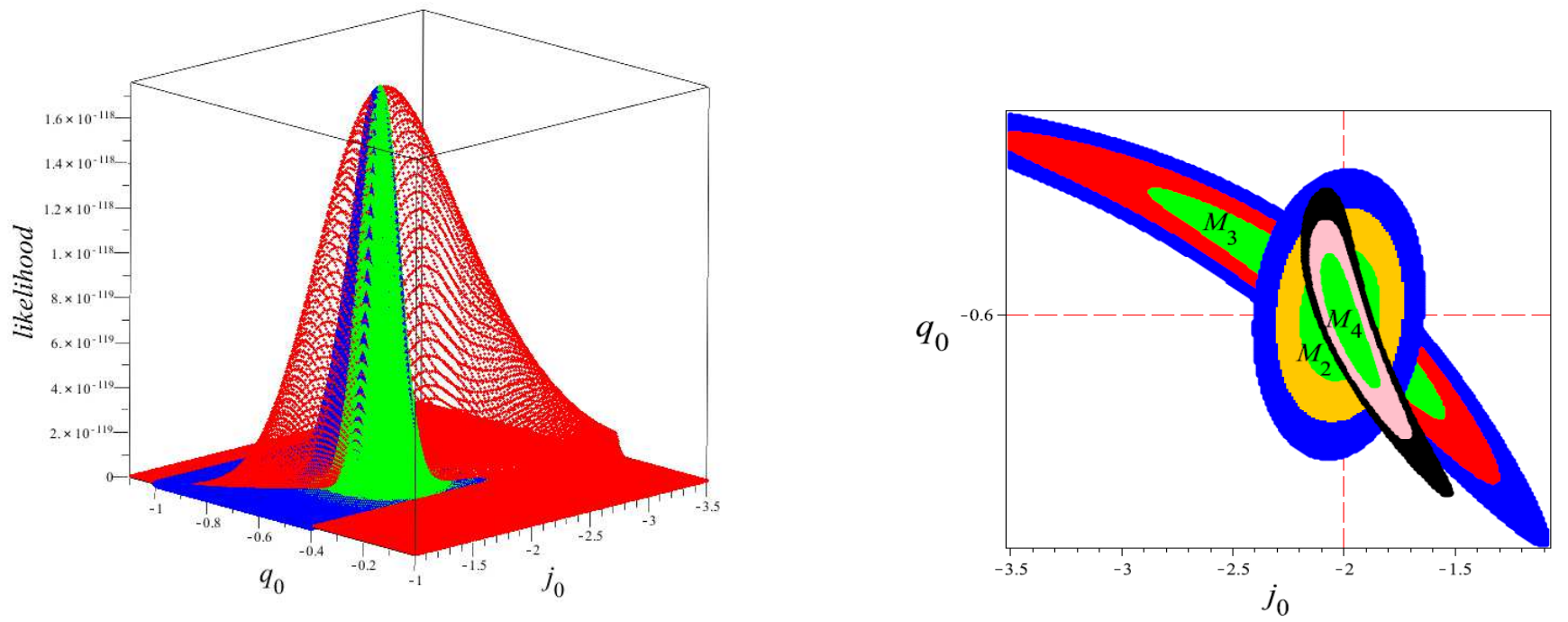

Fig. 4: The 2-dim likelihood and confidence level for parameters $q_{0}$ and $j_{0}$ in $M_{2}, M_{3}$ and $M_{4}$.
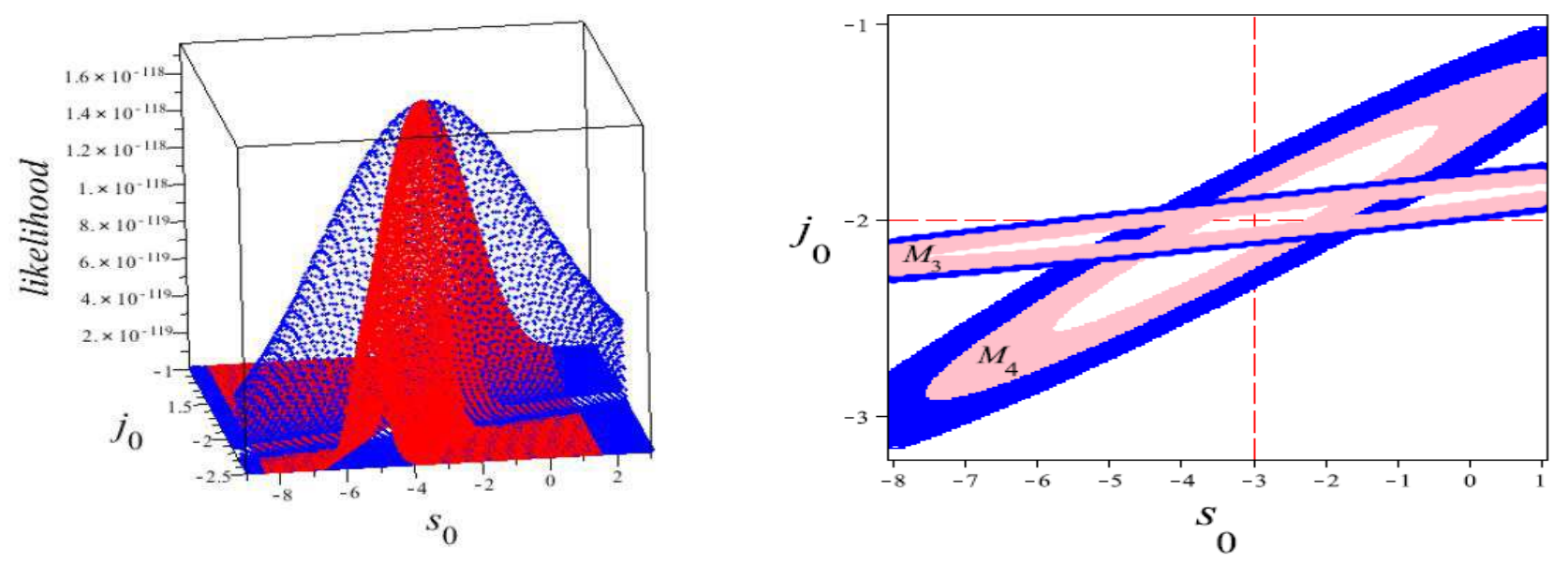

Fig. 5: The 2-dim likelihood and confidence level for parameters $s_{0}$ and $j_{0}$ in $M_{2}, M_{3}$ and $M_{4}$. 

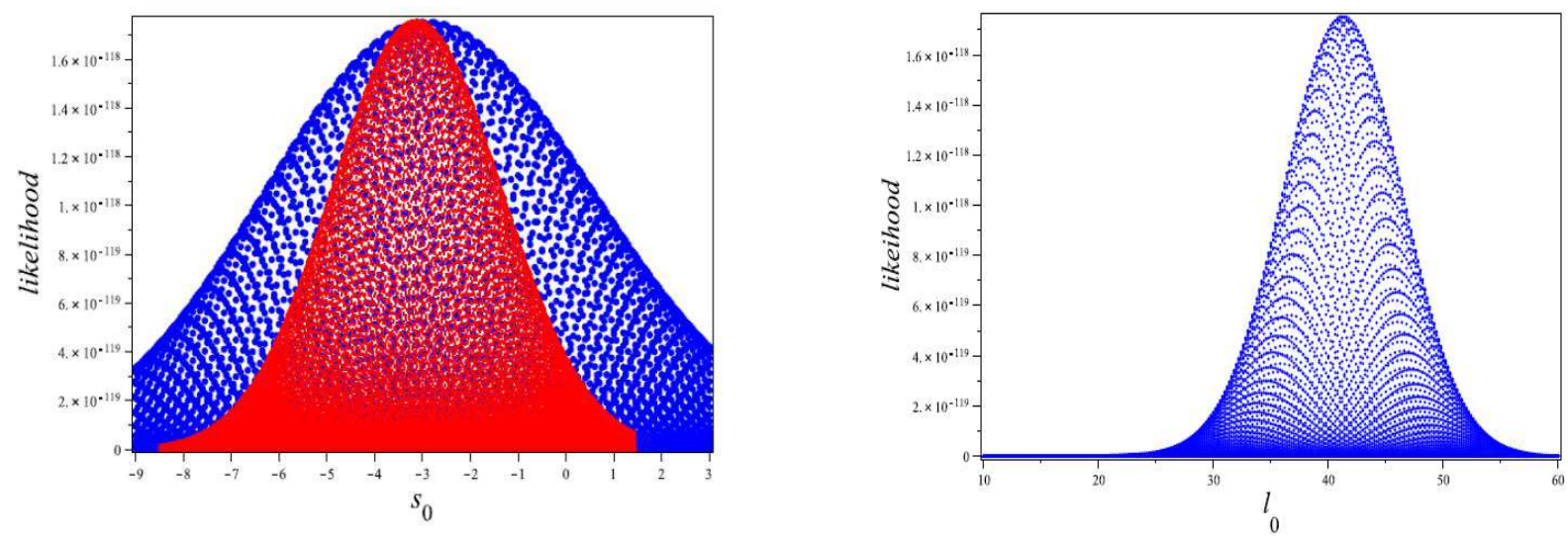

Fig. 6: The 1-dim likelihood for parameter $s_{0}$ and $l_{0}$ in $M_{3}$ and $M_{4}$ cases.

we can find the magnitude of dipole $A$ and direction of preferred axis in galactic coordinates $(l, b)$.

TABLE II: Best-fitted cosmographic parameters in isotropic background

\begin{tabular}{|c|c|c|c|c|c|c|}
\hline \hline model & $h_{0}$ & $q_{0}$ & $j_{0}$ & $s_{0}$ & $l_{0}$ & $\chi_{\min }^{2}$ \\
\hline \hline M1 & $0.687_{-0.0048}^{+0.0048}$ & $-0.204_{-0.12}^{+0.10}$ & - & - & - & 559.3851412 \\
\hline M2 & $0.699_{-0.0045}^{+0.0045}$ & $-0.582_{-0.044}^{+0.06}$ & $-2.05_{-0.11}^{+0.12}$ & - & - & 542.2149085 \\
\hline M3 & $0.6993_{-0.0046}^{+0.0046}$ & $-0.594_{-0.8}^{+0.08}$ & $-2.03_{-0.82}^{+0.53}$ & $-3_{-5}^{+5.2}$ & - & 542.2794916 \\
\hline M4 & $0.6994_{-0.0046}^{+0.0017}$ & $-.6_{-0.09}^{+0.08}$ & $-2_{-0.09}^{+0.1}$ & $-2.9_{-3.1}^{+2.9}$ & $41.2_{-8.2}^{+7.8}$ & 542.2881784 \\
\hline
\end{tabular}

We construct $2.5 \times 10^{4}$ such Monte Carlo datasets and obtain the probability distribution of the dipole magnitude, as well as the corresponding dipole directions.The distribution of the Union2 SnIa datapoints in galactic coordinates along with the Dark Energy dipole direction $(l, b)$ in $1-\sigma$ confidence region for different cases $M_{2}$ to $M_{4}$ are shown in Figs.(7) to (9). Interestingly these directions are very close to each other and point towards $\left(l \simeq 297^{\circ}, b=3^{\circ}\right)$, also the magnitude of the dipole are found to be $|A| \simeq 10^{-3}$. The likelihood distribution of dipole magnitude has been shown in Fig.(10). The results of this work are compared by other studies and is approximately consistent with the results of [47]-[50] and the results of [47]-[50], which have been shown in Fig.( 11). 


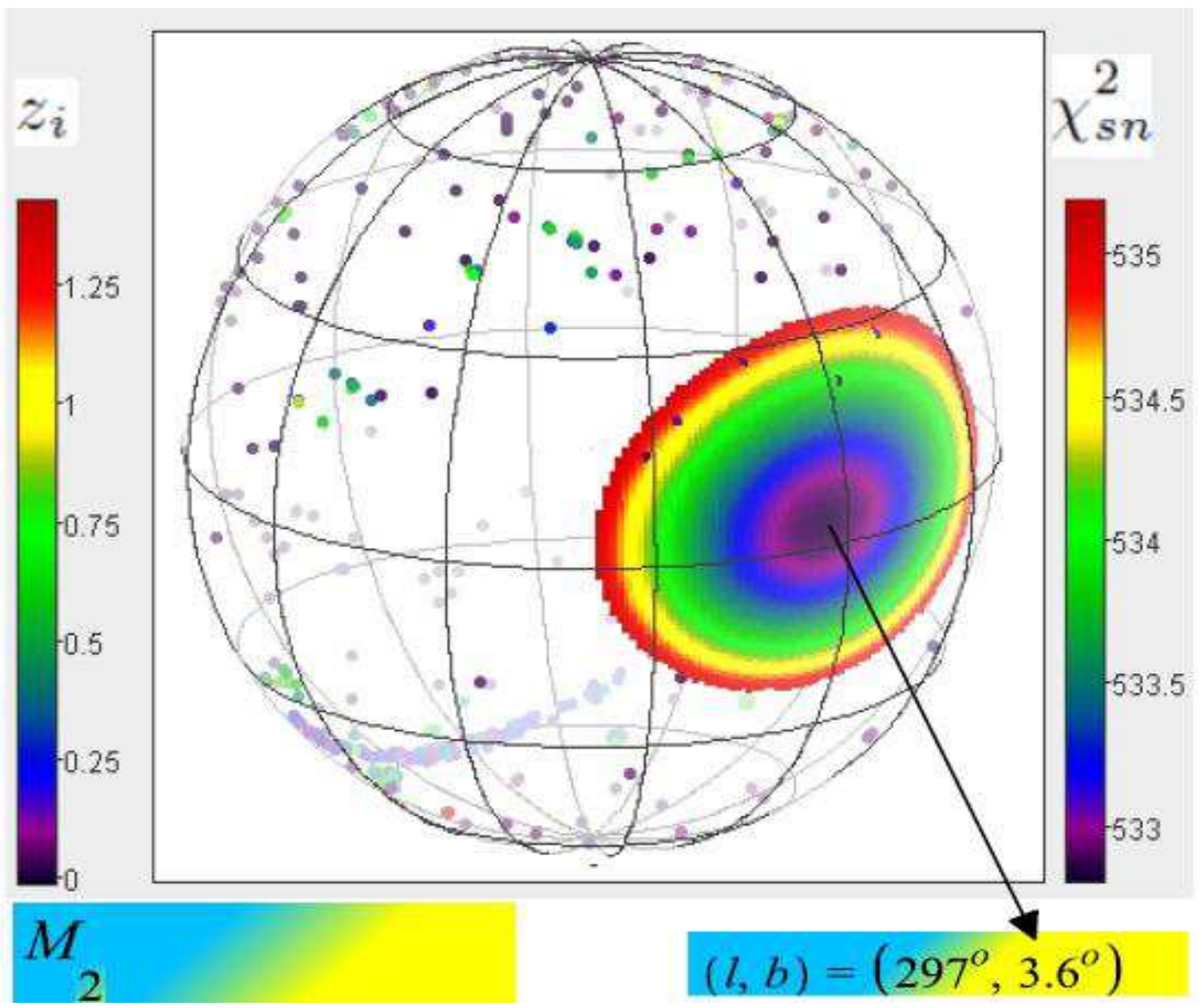

Fig.7: $1-\sigma$ confidence level for parameters $(l, b)$.

SNe Ia samples and the dipolar expansion direction in the galactic coordinates (used Monte Carlo simulation with $2.5 \times 10^{4}$ datasets) 


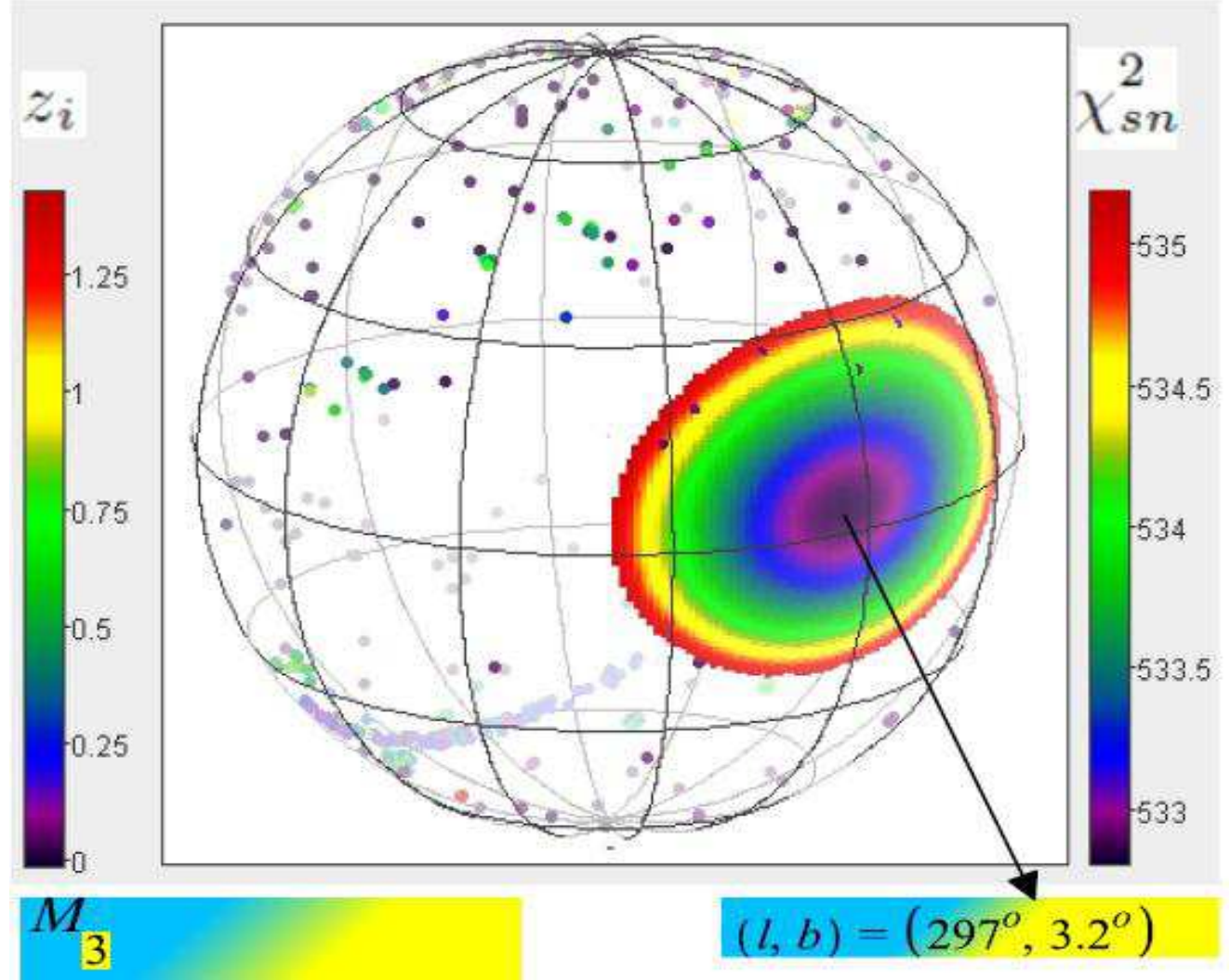

Fig.8: $1-\sigma$ confidence level for parameters $(l, b)$.

SNe Ia samples and the dipolar expansion direction in the galactic coordinates (used Monte Carlo simulation with $2.5 \times 10^{4}$ datasets) 


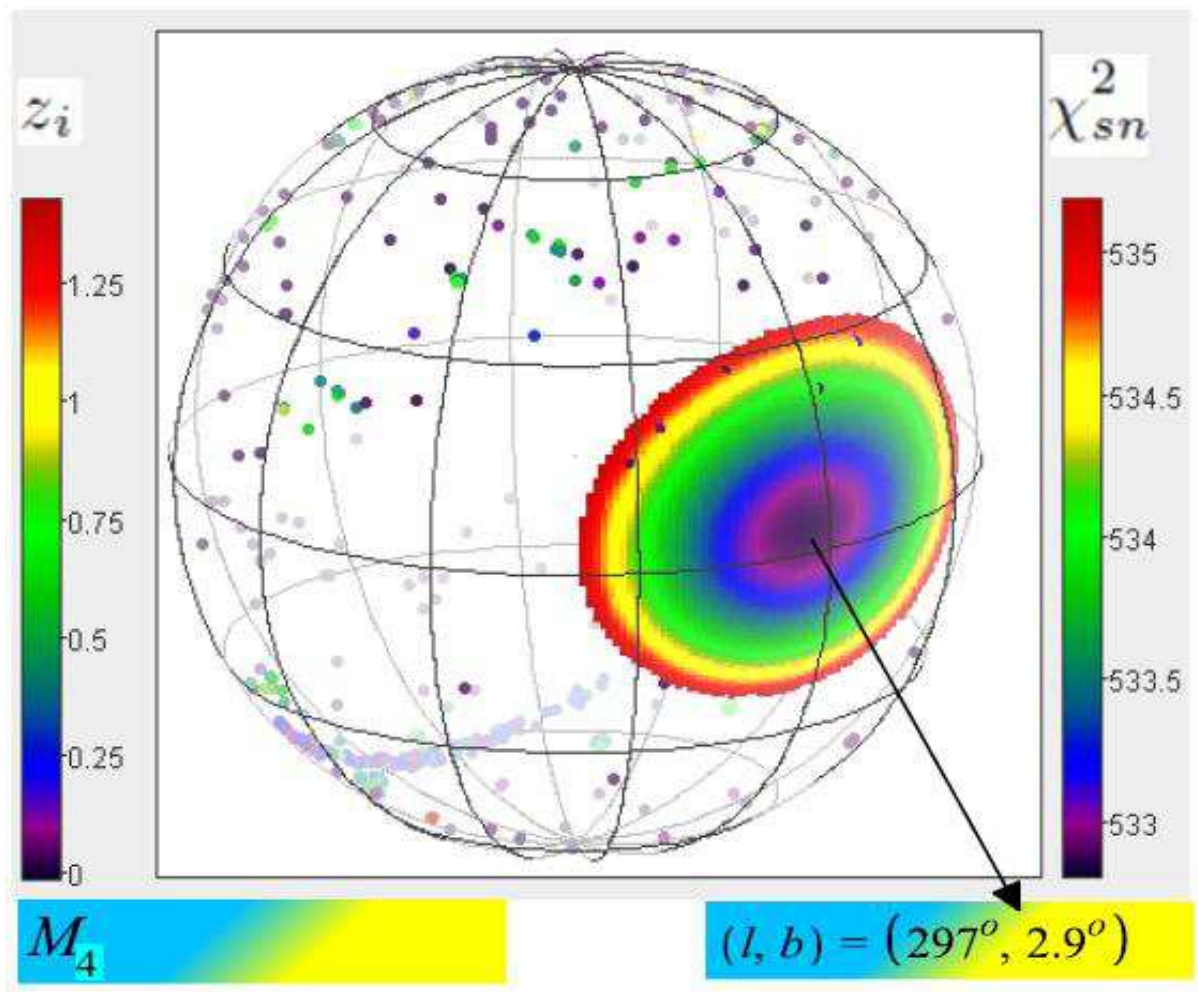

Fig.9: $1-\sigma$ confidence level for parameters $(l, b)$.

SNe Ia samples and the dipolar expansion direction in the galactic coordinates (used Monte Carlo simulation with $2.5 \times 10^{4}$ datasets) 


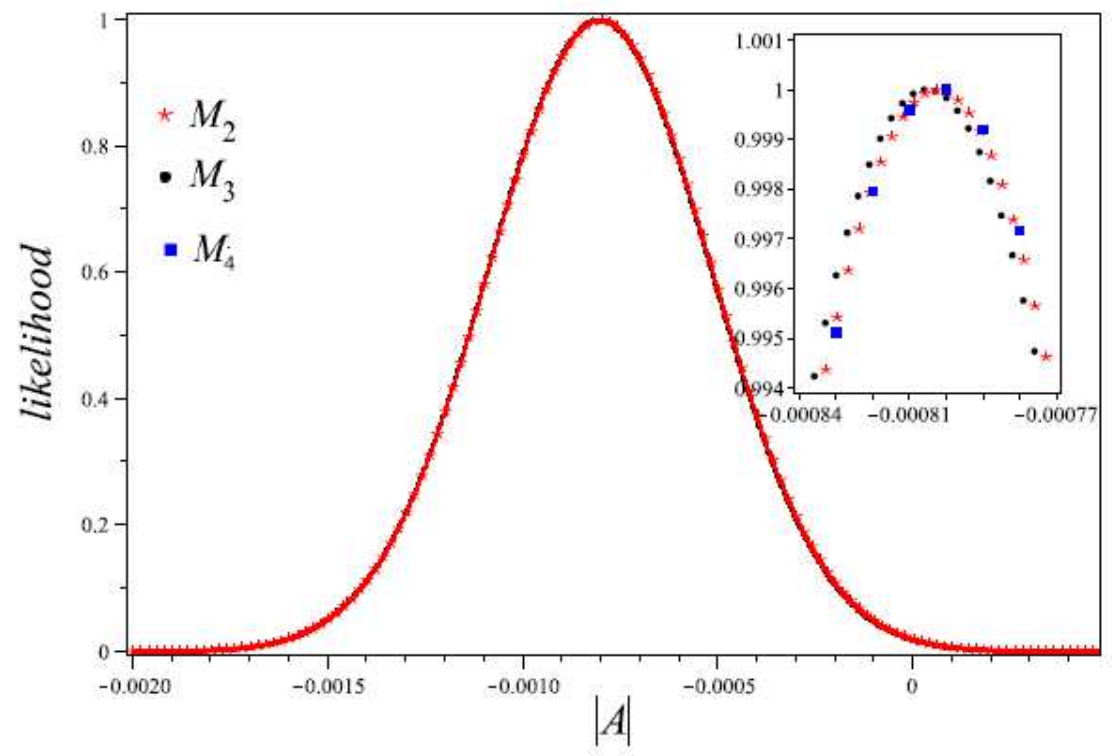

Fig.10:The Anisotropic Magnitude A In The 600 Simulations Follow The Gauss Distribution 


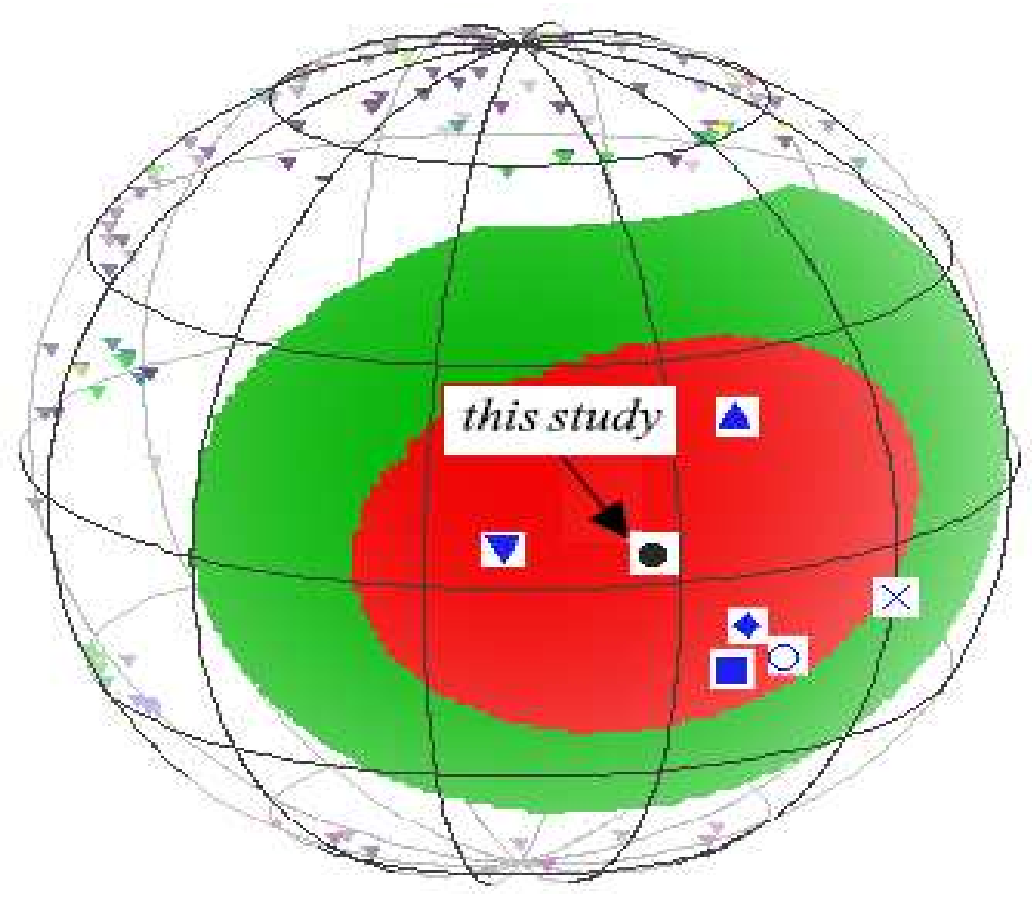

Fig.11: the 1- $\sigma$ and 2- $\sigma$ regions on the Dark Energy dipole direction, which include the results for preferred direction in other models.

direction of preferred axis in galactic coordinate. The point

- denotes our result, namely, $(l, b)=\left(298.7_{+32}^{-32}, 3_{+28}^{-28}\right)$. The results for

preferred direction in other models are presented for contrast. Point $\boldsymbol{\Delta}$ denotes

the result of [33], point $\boldsymbol{\square}$ denotes the result of [38] and [28] ,point $\boldsymbol{\nabla}$ denotes the result of [50], point $\checkmark$ denotes the result of [37],

point $\times$ denotes the result of [47], point $\circ$ denotes the result of [47],

\section{CONCLUSIONS AND DISCUSSIONS}

From some astronomical observations and some the oretical models of the universe, there seemingly exists some evidence for a cosmological preferred axis. In this paper, we study the anisotropic expansion of the universe using type Ia supernovae Union2 sample. The luminosity distance is expanded with model-independent cosmographic parameters as a function of 
redshift $z$ directly. The advantage of this method is that it does not rely on the particular cosmological model.By defining modified redshift $\tilde{z}$ the anisotropic luminosity distance and consequently the transformation matrix $M$ have been obtained. We performed statistical analysis for the $M_{1}, M_{2}, M_{3}$ and $M_{4}$ cases of Matrix $M$,corresponding to the first,second,third and fourth order of Luminosity distance expansion. We found that for $n>2$, the $\operatorname{Model}\left(M_{n}\right)$ is not very sensitive to order of expansion. Thus we cut our analysis for $n>4$. We found the direction of preferred axis for $M_{2}$ as $(l, b) \simeq\left(297_{+34}^{-34}, 3.6_{+28}^{-28}\right), M_{3}$ as $(l, b) \simeq\left(297_{+34}^{-33}, 3.2_{+28}^{-27}\right)$ and $M_{4}$ as $(l, b) \simeq\left(297_{+34}^{-35}, 2.9_{+29}^{-28}\right)$ which are very close to each other. Also these results are compatible with other studies in $(1-\sigma)$ error region[35]-[50]. Also the magnitude of dipole in these cases are very close to each other $\left(A \simeq 10^{-3}\right)$ and other pervious studies. 
[1] E. Komatsu et al. [WMAP Collaboration], Seven-Year Wilkinson Microwave Anisotropy Probe (WMAP) Observations: Cosmological Interpretation, Astrophys. J. Suppl. 192, 18 (2011) arXiv:1001.4538 [astro-ph.CO]].

[2] C. L. Bennett, R. S. Hill, G. Hinshaw, D. Larson, K. M. Smith, J. Dunkley, B. Gold and M. Halpern et al. Seven-Year Wilkinson Microwave Anisotropy Probe (WMAP) Observations: Are There Cosmic Microwave Background Anomalies? Astrophys. J. Suppl. 192, 17 (2011) arXiv:1001.4758 [astro-ph.CO]].

[3] R. Watkins , H. A. Feldman, M. J. Hudson, Mon. Not. R. Astron. Soc. 2009, 392, 743756. Galaxies 2014, 254

[4] M. Kowalski et al. [Supernova Cosmology Project Collab- oration], Astrophys. J. 686, 749 (2008) arXiv:0804.4142[astro-ph]].

[5] R. Amanullah et al., Astrophys. J. 716, 712 (2010) arXiv:1004.1711].

[6] D. Hutsemekers, R. Cabanac, H. Lamy and D. Sluse, Astron. Astrophys. 441, 915 (2005).

[7] D. Hutsemekers, A. Payez, R. Cabanac, H. Lamy, D. Sluse, B. Borguet and J. R. Cudell, ASP Conf. Series 449 (2011) 441.

[8] A. Kashlinsky, F. Atrio-Barandela, D. Kocevski and H. Ebeling, Astrophys. J. 686, L49 (2008).

[9] R. Watkins, H. A. Feldman and M. J. Hudson, Mon. Not. Roy. Astron. Soc. 392, 743 (2009).

[10] G. Lavaux, R. B. Tully, R. Mohayaee and S. Colombi, Astrophys. J. 709, 483 (2010).

[11] C. H. Lineweaver, L. Tenorio, G. F. Smoot, P. Keegstra, A. J. Banday and P. Lubin, Astrophys. J. 470, 38 (1996).

[12] M. Tegmark, A. de Oliveira-Costa and A. Hamilton, Phys. Rev. D 68, 123523 (2003).

[13] P. Bielewicz, K. M. Gorski and A. J. Banday, Mon. Not. Roy. Astron. Soc. 355, 1283 (2004).

[14] C. J. Copi, D. Huterer, D. J. Schwarz and G. D. Starkman, Advances in Astro. 2010, 17 (2010).

[15] M. Frommert and T. A. Enlin, Mon. Not. Roy. Astron. Soc. 403, 1739 (2010).

[16] V. A. Dzuba, V. V. Flambaum and J. K. Webb, Phys. Rev. Lett. 82, 888 (1999).

[17] M. T. Murphy, et al., Mon. Not. R. Astron. Soc. 327, 1208 (2001).

[18] J. K. Webb, J. A. King, M. T. Murphy, V. V. Flam- baum, R. F. Carswell and M. B. Bainbridge, Phys.Rev. Lett. 107, 191101 (2011) arXiv:1008.3907 [astro- ph.CO]]; J. A. King, 
J. K. Webb, M. T. Murphy, V. V. Flambaum, R. F. Carswell, M. B. Bainbridge, M. R. Wilczynska and F. E. Koch, arXiv:1202.4758 [astro-ph.CO].

[19] A. Mariano and L. Perivolaropoulos, Phys. Rev. D 86, 083517 (2012) arXiv:1206.4055 [astroph.CO]].

[20] M. T. Murphy, J. K. Webb and V. V. Flambaum, Mon. Not. R. Astron. Soc. 345, 609 (2003).

[21] J. A. King, et al., Mon. Not. R. Astron. Soc. 422, 3370 (2012).

[22] C. L. Bennett, et al., [WMAP Collaboration], Astrophys. J. Suppl. 192, 16 (2011).

[23] C. L. Bennett, et al., [WMAP Collaboration], Astrophys. J. Suppl. 208, 20 (2013).

[24] P. A. R. Ade et al., [Planck Collaboration], arXiv: 1303.5083 (2013).

[25] Mariano, A. Perivolaropoulos, L. Is there correlation between fine structure and dark energy cosmic dipoles? Phys. Rev. D 2012, 86, 083517.

[26] L. Campanelli, P. Cea, G. L. Fogli and A. Marrone, Phys. Rev. D83, 103503, (2011).

[27] R. G. Cai, Y. Z. Ma, B. Tang, Z. L. Tuo, Constraining the anisotropic expansion of the Universe, Phys. Rev. D 2013, 87, 123522.

[28] R. Cooke, D. Lynden-Bell, Does the Universe accelerate equally in all directions? Mon. Not. R. Astron. Soc. 2010, 401, 14091414.

[29] L. Perivolaropoulos, Large Scale Cosmological Anomalies and Inhomogeneous, Galaxies, 2, 22-61 (2014).

[30] L. Perivolaropoulos, arXiv:0811.4684 [astro-ph].

[31] M. Blomqvist, J. Enander and E. Mortsell, arXiv:1006.4638.

[32] P. Duffett-Smith, 'Practical Astronomy with your Calcu- lator' Cambridge University Press (1989).

[33] I. Antoniou and L. Perivolaropoulos, JCAP 1012, 012 (2010) [arXiv:1007.4347 [astro-ph.CO]].

[34] D. J. Schwarz and B. Weinhorst, Astron. Astrophys. 474, 717 (2007) arXiv:0706.0165 [astro$\mathrm{ph}]$.

[35] R. G. Cai and Z. L. Tuo, JCAP 1202, 004 (2012) arXiv:1109.0941 [astro-ph.CO]].

[36] Benedict Kalus, Dominik J. Schwarz, Marina Seikel, Alexander Wiegand, Astron. Astrophys. 553 (2013) A56.

[37] J. S. Wang, F. Y. Wang, Mon. Not. R. Astron. Soc. 443,1680-1687 (2014).

[38] Xiaofeng Yang, F. Y. Wang, Zhe Chu, Mon. Not. Roy. Astron. Soc. 437: 1840-1846, (2014).

[39] Li X., Lin H.-N., Wang S., Chang Z., EPJC, 73, 2653, (2013) 
[40] Zhe Chang, Ming-Hua Li, Sai Wang, Phys. Lett. B 723, 257, (2013).

[41] De-Chang Dai, William H. Kinney and Dejan Stojkovic, JCAP 1004, 015, (2011).

[42] L. Hui and P. B. Greene, Phys. Rev. D73, 123526 (2006), astro-ph/0512159.

[43] M. Sasaki, Mon. Not. Roy. Astron. Soc. 228, 653 (1987).

[44] N. Sugiura, N. Sugiyama, and M. Sasaki, Progress of Theoretical Physics 101, 903 (1999).

[45] T. Pyne and M. Birkinshaw, Mon. Not. Roy. Astron. Soc. 348, 581 (2004), astro-ph/0310841.

[46] C. Bonvin, R. Durrer, and M. A. Gasparini, Phys. Rev. D73, 023523 (2006), astro-ph/0511183.

[47] Z. Chang, M. H. Li, X. Li S. Wang, Eur. Phys. J. C 74, 2821, (2014).

[48] Z. Chang, M. H. Li, X. Li S. Wang, Mod. Phys. Lett. A 29, 1450067, (2014)

[49] Z. Chang, M. H. Li, X. Li, S. Wang, Eur. Phys. J. C 73, 2459 (2013).

[50] H. A. Feldman, R. Watkins, M. J. Hudson, Cosmic flows on 100 Mpc/h scales: Standardized minimum variance bulk flow, shear and octupole moments. Mon. Not. R. Astron. Soc. 407, 2328, (2010).

[51] H. A. Feldman, R. Watkins and M. J. Hudson, Mon. Not. Roy. Astron. Soc. 392, 756 (2010) arXiv:0911.5516 [Unknown]].

[52] S. Capozziello, V. F. Cardone, V. Salzano Phys. Rev. D78, 063504, (2008).

[53] C. Cattoen, M. Visser, Class. Quant. Grav. 24, 5985, (2007).

[54] C. A. P. Bengaly Jr, JCAP 04 (2016), 036, arXiv:1510.05545 [astro-ph.CO]; K. Tomita, Prog. Theor. Phys. 105, 419, (2001), arXiv:astro-ph/0005031; M. L. McClure, C. C. Dyer, New Astron, 12, 533, (2007), arXiv:astro-ph/0703556; L. Zaninetti, Advanced Studies in Theoretical Physics (2014), 8, 599, arXiv:1409.8100 [astro-ph.CO]; A. E. Romano, S. A. Vallejo, arXiv:1403.2034 [astro-ph.CO]. 